\title{
SECLOUD: Source and Destination Seclusion Using Clouds for Wireless Ad hoc Networks
}

\author{
Razvi Doomun, Thaier Hayajneh, Prashant Krishnamurthy, and David Tipper \\ University of Pittsburgh \\ Pittsburgh, PA, USA \\ Emails: \{mrazvi, hayajneh, prashant, dtipper\} @ sis.pitt.edu
}

\begin{abstract}
The privacy of communicating entities in wireless ad hoc networks is extremely important due to the potential of their being identified and subsequently subjected to attacks (e.g., in military networks). Previously, the random walk and fractal propagation schemes have been proposed to address privacy of source and destination nodes in ad hoc or sensor networks. Entropy of packet transmissions has been used as the metric for comparison. In this paper, we show that under a global attacker that can eavesdrop on the overall data transmissions and count them, neither of these approaches provide sufficient privacy when the attacker can visualize the transmissions and infer contextual information. Moreover, we show that the entropy is not a useful metric in such a case. We propose SECLOUD: Source and Destination Seclusion using Clouds to obfuscate the true source/destination nodes and make them indistinguishable among a group of neighbor nodes which works well even under network-wide traffic visualization by a global attacker.
\end{abstract}

\section{INTRODUCTION}

Malicious traffic analysis and privacy attacks against source and destination nodes in ad hoc networks are passive and difficult to detect in large wireless ad hoc networks [1]. Moreover, the disclosure of contextual information about network traffic patterns can be devastating in privacy-sensitive application scenarios. For example, in a military ad hoc network in the battlefield, command centers could be communicating with each other through an ad hoc network of intermediate nodes. Analysis of traffic in such an environment may reveal the locations of command centers which will enable the adversaries to launch targeted cyber or physical attacks on them. Hence, it is more critical to hide the location of the source as well as ensure the privacy of destination for quasi-stationary nodes in ad hoc networks. Spreading the network traffic among several paths is also a first step countermeasure against traffic analysis attacks. Different methods have been proposed in the literature for defending against traffic analysis and location privacy related attacks [2]-[5]. We note here that most of the research work assumes that all packets are encrypted link-by-link, padded to hide potential packet types through size, and use anonymous routing schemes to avoid detection of routes during route setup. Further, energy constraints and overhead are typically not factored because obfuscation requires transmission of dummy packets (discussed later).

However, most existing solutions to protect privacy of source or destination nodes do not consider the possibility of attackers having a complete view of the network traffic, in which case they fail to work well. In this paper we address the problem of secluding source/destination nodes in the presence of an attacker with complete view of the network topology and traffic. In wireless ad hoc networks, attackers can be broadly classified as either local or global. A local attacker can generally eavesdrop on transmitted packets around one node at a time and it does not know the overall network topology, whereas a global attacker is able to visualize the overall network topology and is capable of network-wide traffic rate monitoring and time-correlation attacks. Networkwide rate monitoring attack involves counting the number of transmitted/received packets around every node in the network, while time-correlation attacks involve finding the communication patterns by analyzing latencies between packet transmissions around nodes in the network.

In this work, we propose a simple and efficient technique for source/destination privacy against such a global adversary. The proposed protocol, SECLOUD: Source and Destination Seclusion using Clouds, hides the source and destination nodes in a group of nodes (called "cloud") that are indistinguishable. The performance of SECLOUD is evaluated assuming a global attacker model capable of using network-wide traffic visualization. We compare SECLOUD with two commonly used privacy techniques, Random Walk and Fractal Propagation [2], [6]. SECLOUD provides better privacy than either scheme in the presence of a global traffic visualization attack. We show that entropy, which is a common privacy metric, is not always an adequate measure of anonymity strength and also quantify the privacy level using anonymity and unlinkability metrics. Our simulations show that SECLOUD has a lower overhead compared to the fractal propagation and random walk schemes.

The rest of the paper is organized as follows. Section 2 describes related work on popular privacy techniques. The attacker model, network assumptions, and privacy metrics are presented in Section 3. The details of SECLOUD protocol are explained in Section 4. Section 5 presents the simulation set-up and results discussion. Section 6 concludes the paper.

\section{RELATED WORK}

Previous works on privacy mechanisms for ad hoc wireless networks provide some level of protection against attackers by thwarting traffic analysis attacks and misleading attackers with randomized or fake traffic [1]-[4], [6]-[8]. However, several of these approaches deal with location privacy in wireless sensor networks where the entity to be protected is the sink node [2], [5] or the sensing sources only [6]-[8].

Random Walk: Kamat et al. [7] and Ozturk et al. [9] proposed similar techniques based on a random walk approach for source location privacy in sensor networks against an 
external local adversary. Xi et al. [6] developed an improved random walk approach that starts from both source and sink. The random walk operates by forwarding packets probabilistically from the source through an unpredictable route (with extra hops than the shortest path) to reach the destination [6]. Using erratic per-packet route is very effective in resisting rate monitoring and traffic analysis attacks by adversaries monitoring a limited set of nodes. The random walk increases the time to trace the source location and thus the source can communicate privately with the destination for a longer time. However, it is hard to completely obfuscate the general trend of the traffic flow towards the destination location.

Fractal Propagation: Deng et al. [2] have proposed the fractal progation technique to counteract local rate monitoring and correlation attacks against location privacy in sensor networks. Fractal propagation overcomes one of the drawbacks of the random walk scheme by introducing fake packet spreading to combat time correlation attacks [2]. When a neighbor node overhears a packet transmission, it probabilistically generates a fake packet with $k$-hop lifetime and forwards it to one of its neighbors. The propagation distance $k$ of the fake packets causes network traffic to appear spread out along different routes resulting in tree-like transmission paths that are more diffused than the random walk method. However, fractal propagation cannot totally obfuscate traffic patterns near the source and destination area, because all real packets diverge from the source node and converge towards the destination node. Thus, eavesdropping by a global attacker which can visualize the network-wide traffic can expose the source and destination regions as we show later.

In [10], a path confusion algorithm is used to increase source location anonymity against a local adversary model. Mehta et al. in [3] proposed a privacy scheme under a global external attack model by hiding the real source among $k-1$ fake sources simulating the mobility pattern of real sources in sensor network. In [8], under the global attack model, the authors proposed statistically strong source anonymity by employing network-wide dummy messages to achieve global privacy. In [4], the authors introduce a similar approach with carefully chosen dummy traffic to hide the real event sources in combination with mechanisms to drop dummy messages to prevent explosion of network traffic. Some sensor nodes act as proxies that proactively filter dummy messages on their way to the base station destination. The amount of dummy traffic, and location and number of fake sources are important factors that determine the effectiveness of the aforementioned privacy mechanisms.

\section{Models AND Assumptions}

\section{A. Network model}

In this work, we consider an ad hoc network with nodes being distributed in a grid like manner (or randomly). Our assumptions are very similar to most related work in the literature. The IEEE 802.11 standard for physical and MAC layers is assumed and nodes do not use RTS or CTS to avoid revealing communication peers. All the MAC and routing protocol messages are assumed to be encrypted so that no leakage of information occurs to the adversary. The nodes'
MAC address, IP address and node IDs will also be hidden and not advertised. In [11] the authors used short-lived disposable MAC addresses to prevent the real node IDs from being revealed to adversaries. A similar technique will be assumed in this paper to avoid any identification of nodes. We assume the existence of a key management protocol that can distribute pair-wise keys between nodes or public-private key pairs for each node [12], [13]. Any of these schemes can be used to set up pairwise keys and authenticate nodes' relationship, but we omit such details here in this paper. Each packet is encrypted and authenticated so that an adversary cannot decrypt or modify the contents of an eavesdropped packet transmission. All packets are transmitted in the same format and have same length (by padding or fragmenting). Finally, route discovery communications are assumed to be anonymous using any of the anonymous routing protocols such as in [14] [18]. An anonymous routing protocol allows neighbor nodes to authenticate each other without revealing their identities. For example, in [14] the anonymous neighbor authentication is based on dynamically changing pseudonyms of nodes instead of their real identifiers or MAC addresses. Anonymous route discovery and data forwarding employ pairwise shared link identifiers between neighbor nodes which are created and established during neighborhood authentication.

\section{B. Attack Model}

An external, global, and powerful attacker model is assumed in this paper that has combined capabilities of different existing attack models as described in [2], [3], [19]. The attacker has complete knowledge of network topology and can keep statistical measurements for all of the network traffic. We assume that the global attacker can perform rate monitoring and time correlation attacks for all traffic in the network (which is a stronger attack than corresponding ones assumed elsewhere). The attacker will visualize all transmitted/received packets in the network and determine the traffic density on every link in the network. However, the attacker is passive and cannot compromise nodes in the network. A possible method for this attack is by deploying an overlay network with several malicious nodes simply to sense traffic from the given ad hoc network, similar to the idea in [3]. These nodes can collect information and collaborate with a centralized entity using a different band. We investigate the ability of privacy techniques to withstand this powerful global attacker. We consider a single source and destination in the network that makes it easiest for an attacker to identify them, but SECLOUD can be deployed with multiple sources and destinations as well.

\section{Privacy Evaluation Metrics}

We analyze the performance of SECLOUD, random walk and fractal propagation using the metrics - anonymity, unlinkability [19], and entropy.

1) Anonymity: The level of anonymity $\lambda$ is defined as the probability that a node of interest is incorrectly identified in an anonymous group [19]. If a node is hidden among $A$ nodes that have the same behavior, then the level of anonymity is $\lambda=1-(1 / A)$. If the attacker estimates that the sourcedestination pair is in the anonymity sets $A_{1}, A_{2}, \ldots A_{n}$, where 
$|A|$ is the size of a set $A$ and $n$ is number of sets, then, the anonymity level for this node of interest is given by $\lambda=1-\left\{1 / \mid A_{1} \cup A_{2} \cup \ldots \cup A_{n}\right\} \mid$. Thus, the anonymity level of a node (source/destination in our case) depends on the number of nodes in the anonymous zone, which is the cloud area in SECLOUD.

2) Unlinkability: We employ a 3-D graph of transmitted data around nodes to determine whether or not a global attacker can visualize the existence of communication between a source and destination. More powerful edge detection algorithms are also possible (we show one example). Details are provided in Section V.

3) Entropy: Entropy, a common metric for quantifying uncertainty in information theory, is often used as a privacy measure [2]. It is a scalar number that does not capture the amount of contextual information, such as paths analysis, that disclose hints about the location of source and destination nodes.

\section{The SECLOUD PROTOCOL}

The general idea of SECLOUD, is to seclude the source and destination node locations within a cloud of irregular shape that is constructed using its neighboring nodes. The details of this protocol are explained next.

First, the source node $S$ broadcasts a hello message to discover all its one-hop neighbors $N(1, i)$ for $i=1,2, . . m$, where $m$ is the total number of neighbor nodes. Then, the nodes in $N(1, i)$ discover their respective neighbors $N(2, i)$ which are two-hops away from node $S$. Consequently, source node $S$ constructs the list: $N(1, i), N(2, i), N(3, i) \ldots N(k, i)$, where $N(k, i)$ is the set of $k^{t h}$ hop neighbors of node $S$. This initialization process of neighbor discovery is done periodically by all nodes in the network. This will ensure that the attacker cannot determine which of the nodes performing the initialization will be the source.

Let the cloud region be of maximum width $k$ hops from the source. For e.g, with $k=3$, source node $S$ will randomly select a number of nodes, $B$, such that $B \subseteq$ $\{N(1, i) \cup N(2, i) \cup N(3, i)\}$. This enables reducing overhead compared to schemes that use dummy transmissions everywhere or by every node and keep the region irregular - the source cannot be predicted to be in the center of the cloud for example. The nodes in $B$ will be marked as pseudosources in the cloud and are requested to transmit encrypted dummy packets at a rate similar to the source transmission rate and to forward real packets when available from source to delegated sources (see next). Dummy packets are simply dropped. The destination node $D$ which also does the same initialization procedure will construct a cloud. Note the size of the source and the destination clouds can be different by using different values of $k$ for each, depending on the privacy strength needed on each side.

Node $S$ randomly selects one or more nodes from the set $B$ to act as delegated sources. Similarly $D$ randomly selects one or more nodes in its cloud to act as delegated destinations. We consider the case of one delegated source/destination as a special case in Section V. Delegated sources find routes to delegated destinations using a suitable anonymous routing protocol (e.g., see [14]). In any case, local broadcasts/relays from $S$ to the delegated sources and from the delegated destinations to $D$ ensure delivery of data locally. Nodes in $B$ are picked such that there is connectivity between $S$ and the delegated sources. A similar strategy is employed in the destination cloud.

With the above set-up, SECLOUD achieves source/destination privacy in a local regions. In single source-destination scenario, the global attacker will have to guess the source or the destination from the set of nodes $B$ which is the cloud size. To improve privacy, SECLOUD can create fake sources and fake destination clouds as well. The fake sources/destination clouds will behave in a manner similar to the real $S$-D clouds and will communicate with each other. In the best case, paths between fake source/destination clouds will intersect the real traffic flow. Fake clouds increase the likelihood of misleading the attacker who may attack (e.g., by jamming) the fake regions instead of the real regions. When there are multiple sources and destinations with different traffic paths crossing each other in the same time period, fake clouds may be omitted. Nodes in the real source-destination clouds and all the nodes in the fake source-destination regions will need to be loosely synchronized so that the global attacker observes packet transmissions in the same time period.

\section{Evaluation}

We simulated a large network of 400 nodes distributed in an area of $2000 \mathrm{~m} \times 2000 \mathrm{~m}$ with average node degree between 7 and 8. We employed the Quasi-Unit disk graph (Q-UDG) connectivity [20] with some percentage of perturbations and random distribution introduced for grid network topologies. The coordinates of each node $x$ and $y$ were randomly chosen using uniform random variables in the ranges $\left(x-p x_{i}, x+p x_{i}\right)$ and $\left(y-p y_{i}, y+p y_{i}\right)$, respectively, where $p$ is the perturbation parameter and $x_{i}$ and $y_{i}$ are the spacing between the nodes in the $x$ and $y$ directions, respectively $\left(p=0.2\right.$ and $x_{i}=y_{i}=$ $m \times 100$ for integral $m$ were assigned for all our simulations). For random node distribution, the coordinates of the nodes $x$ and $y$ were randomly and independently chosen in the range from 0 to $2000 \mathrm{~m}$. After the nodes are distributed, the QUDG connectivity model and transmission range is used to determine the network topology. A link exists between two nodes if the inter-nodal distance $d$ is less than $\alpha R$, where $R$ is the transmission range of the node and $\alpha$ is the Q-UDG factor $(0 \leq \alpha \leq 1)$. In our simulation we set $\alpha=0.2$. For distances $d$ greater than $R$, there is no link connectivity. However, for $\alpha R \leq d \leq R$, the link will exist with probability $(R-d) /(R-$ $\alpha R)$. The source node is randomly chosen from the region $(100 m \leq \mathrm{x} \leq 900 m)$ and $(100 m \leq \mathrm{y} \leq 900 m)$ and destination node is randomly selected from $(1100 m \leq \mathrm{x} \leq 1900 \mathrm{~m})$ and $(1100 m \leq \mathrm{y} \leq 1900 \mathrm{~m})$ of the network. The source sends 5000 real packets in a time window of $T$ seconds. All simulations are repeated 15 times and results are averaged for each tested scenario.

Metrics: If node $i$ transmits $u_{i}$ packets and a total of $V$ packets were transmitted in the network in time $T$, the fraction of packets sent by $i$ is $p_{i}=u_{i} / V$ and the entropy is defined 
as $H=-\sum_{i} p_{i} \log _{2} p_{i}$. We determine unlinkability and anonymity as follows. To visualize linkable communications, the attacker can simply plot a 3-D graph of the number of packets transmitted by each node in time $T$ with the approximate location of each node. A second approach is to convert this information into an image and use an edge detection algorithm to reveal source/destination locations as well as the communication route. We show an example of the Canny edge detection algorithm [21], which is a popular image processing approach in computer vision. The algorithm first smoothes the image to eliminate noise pixel intensities and finds the image gradient to highlight regions with high spatial derivatives. It then tracks along these regions and suppresses any pixel that is not at the maximum (non-maximum suppression). The gradient matrix is further reduced by hysteresis thresholding. Finally, to better quantify linkability, the attacker will sample $n$ of the nodes that have the highest number of packets transmitted in $T$ and computes the average value $U$ of packets transmitted. Then nodes that transmit at least $\beta U$ packets are marked where $0<\beta<1$. A graph of nodes, the number of packets transmitted and the marked nodes is used to determine possible communication paths, sources, and destinations. We pick $n=10$ in our simulations. The values of $n$ and $\beta$ will create sharp or fuzzy boundaries in the graph. Based on these boundaries, we count the size of $|A|$ - the number of nodes within which the source and destinations are hidden. In the case of random walk we do not include the nodes at the boundary of the network in picking $n$ or marking nodes, as this would cause bias due to packet transmissions bouncing back.

\section{A. Random Walk Technique}

With random walk, [2], [7], packets are forwarded in a random fashion from one hop to the next until they reach the destination. The route taken by each packet from source to destination is unpredictable. A probability $\left(0.5 \leq P_{r} \leq 1.0\right)$ is used at each hop to decide how random the forwarding is. If $P_{r}=1.0$, there is no randomness and the packet is sent to next hop node on the shortest path to the destination.

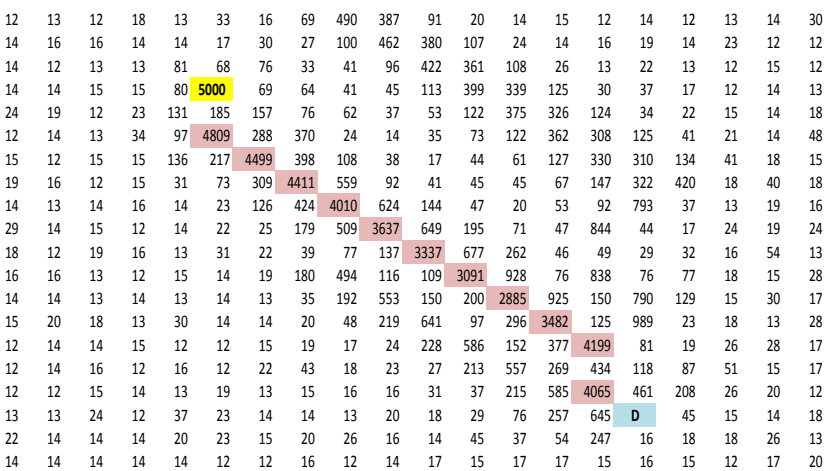

Fig. 1. Random Walk $P_{r}=0.9$ - marking nodes

In Fig. 1, a matrix of the number of packets $u_{i}$ transmitted by each node $i$ is shown for random walk with $\operatorname{Pr}=0.9$ (sample of one simulation). Nodes are not located exactly as shown but their relative positions are maintained in the matrix. The source node $S$ (yellow color) sends 5000 packets to destination node $D$ (blue color). Using $n=10, \beta=0.5$, we find $\beta U=2072$ and mark all nodes transmitting more than $\beta U$ packets. As shown in in Fig. 1, the entire route is revealed. The edge node at the source is even more apparent as it will have a global maximum (highest traffic density node). The destination is one-hop away from the next highest traffic node with packet count 4065 .

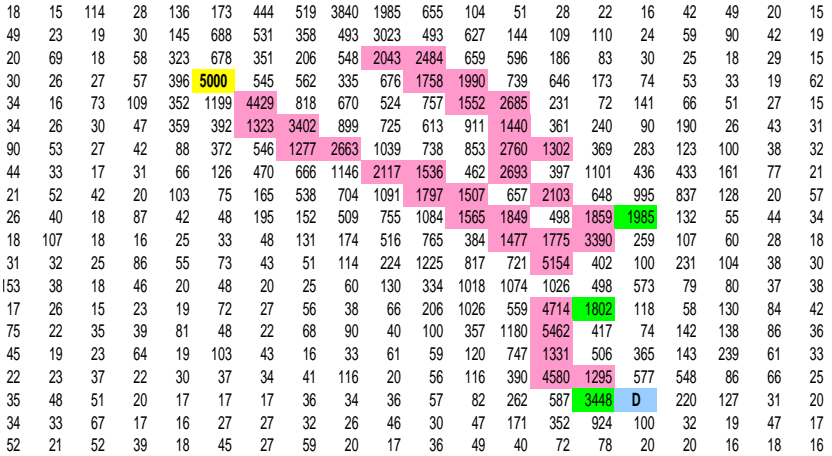

Fig. 2. Random Walk $P_{r}=0.7$ - marking nodes

In Fig. 2, we reduce $P_{r}$ to 0.7 and repeat the process. In this case $(n=10, \beta=0.5, \beta U=2130)$, yields a fuzzy region. We reduced $\beta$ to 0.3 resulting in $\beta U=1278$ and a sharper set of paths is obtained as shown in Figures 2 and 4. The privacy is increased since more than one communication path appears to exist. The destination node can be guessed to be near three nodes with $u_{i}$ 's 1985,1802 and 3448 and destination anonymity is $2 / 3$ (i.e., $|A|=3$ ). The source node packet count is still a global maxima in its area and can still be detected.

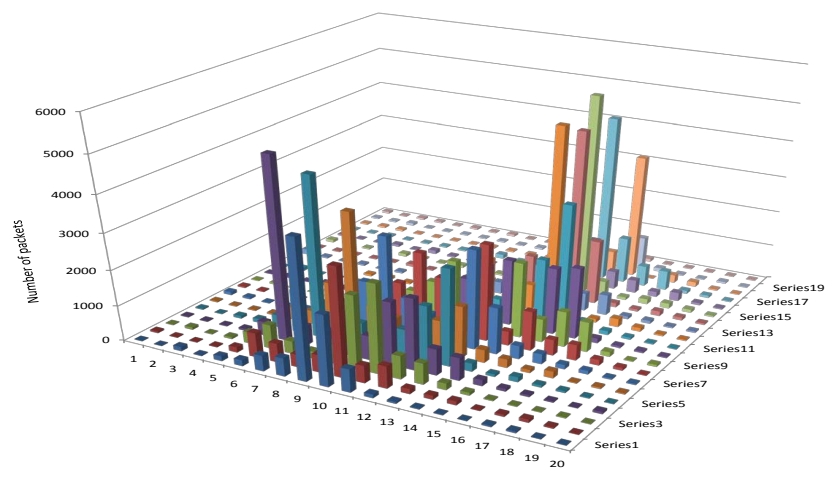

Fig. 3. Random Walk $P_{r}=0.7-3-\mathrm{D}$ graph of $u_{i}$

The 3D traffic graph for this case is shown in Fig. 3. The output of the edge detection algorithm is shown in Fig. 4. The same conclusions can be extracted by the global attacker from these figures. We omit some of these graphs for subsequent privacy techniques (fractal propagation and SECLOUD).

The overhead cost with random walk (average from 15 simulations) is shown in Table I for different values of $P_{r}$. The average path length to reach the destination and the corresponding computed entropy values are also shown in the same table. The transmission overhead (TO) is the ratio of total number of packets transmitted that with the shortest single 


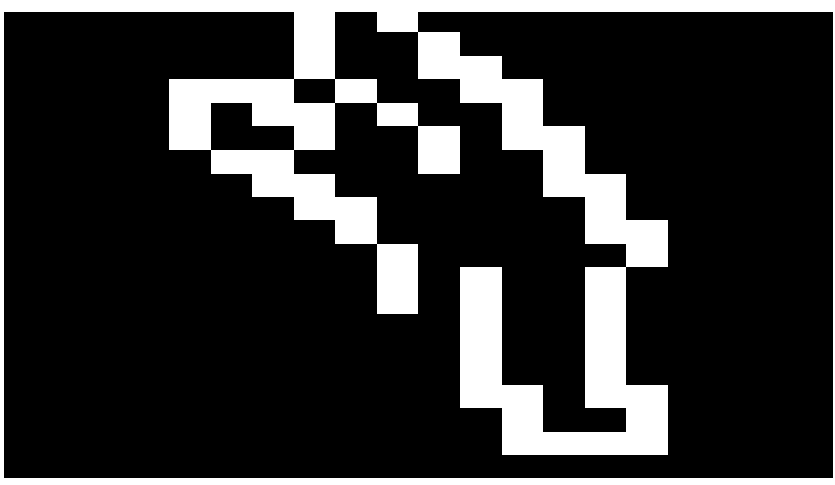

Fig. 4. Random Walk $P_{r}=0.7-$ Edge Detection

\begin{tabular}{|c|c|c|c|}
\hline$\left(1-P_{r}\right)$ & Av. path length & TO & Mean Entropy \\
\hline 0.5 & 60.6 & 4.66 & 7.222 \\
\hline 0.4 & 39.5 & 3.04 & 7.266 \\
\hline 0.3 & 30.4 & 2.34 & 6.853 \\
\hline 0.2 & 25.7 & 1.98 & 6.854 \\
\hline 0.1 & 15.0 & 1.15 & 6.262 \\
\hline
\end{tabular}

TABLE I

RW PROTOCOL

path transmission from a fixed source to a fixed destination 13 hops away. The transmission overhead can be as high as $466 \%$ with for $P_{r}=0.5$. Even with $P_{r}=0.7$, the overhead is $234 \%$ and the anonymity and unlinkability is not adequate. Although, the entropy values increased with decreasing $P_{r}$, they provide little information about the achieved privacy.

\section{B. Fractal Propagation Technique}

In fractal propagation, neighbor nodes along a forwarding path from source to destination generate fake packets with probability $P_{\text {fake }}$, and the fake packets are propagated $K$ hops by successive neighbor nodes randomly selected. A higher $\left(P_{\text {fake }}, K\right)$ is expected to enhance communication privacy with extra overhead.

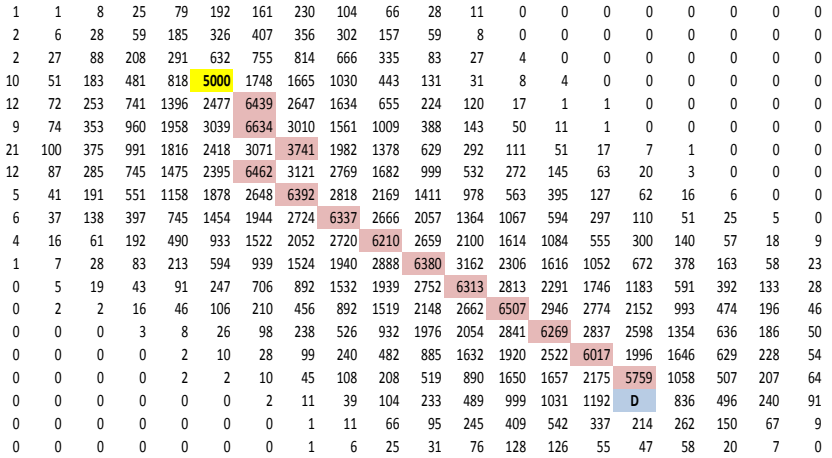

Fig. 5. Fractal Propagation $P_{f a k e}=0.1, K=5$ - marking nodes

The results of traffic visualization for one simulation sample by marking nodes $(n=10, \beta=0.5, \beta U=3197)$ is shown in Fig. 5 for $P_{\text {fake }}=0.1, K=5$. We can identify a single source node (yellow color) transmitting 5000 packets to the destination node 13-hops away, denoted by cell $D$ (blue color).

The 3D traffic graph shown in Fig. 6, illustrates a similar result where the peak traffic nodes are aligned. The anonymity

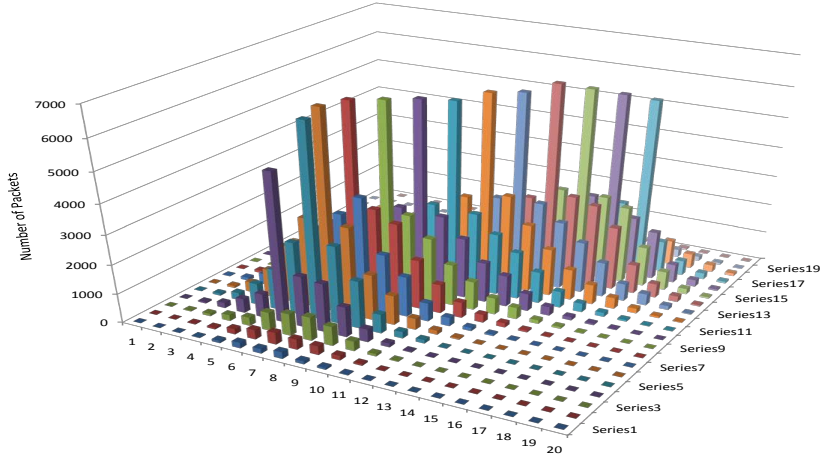

Fig. 6. Fractal Propagation $P_{\text {fake }}=0.1, K=5-3$-D graph

is zero since the source-destination locations are both distinguishable by a global attacker. The destination node is just 1-hop away from the node with packet count 5759 .

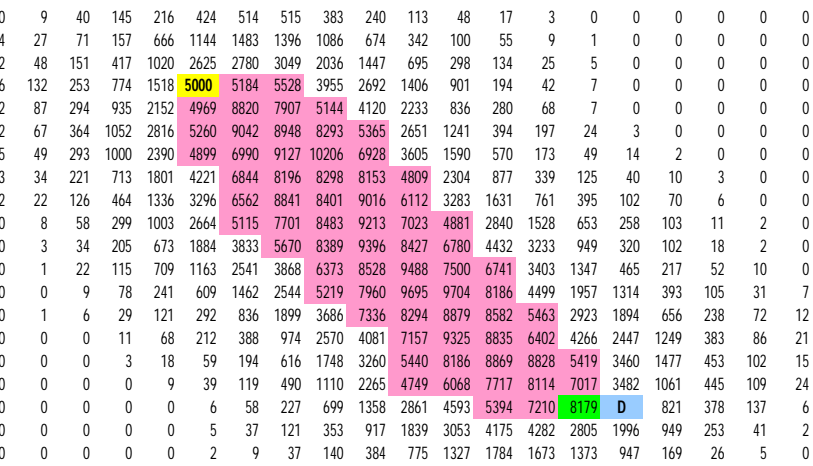

Fig. 7. Fractal Propagation $P_{\text {fake }}=0.3$

In Fig. 7, for the same source and destination nodes, a higher probability of fake packet generation $\left(P_{\text {fake }}=0.3\right)$ is used with fractal propagation. The source and destination nodes can both still be located using the same methodology (for $n=10$, $\beta=0.5$, in this case $\beta U=4710$ ).

\begin{tabular}{|c|c|c|c|}
\hline$P_{\text {fake }}$ and $K$ values & TO & \# Fake Paths & Mean Entropy \\
\hline$P_{\text {fake }}=0.1, K=5$ & 4.75 & 3750 & 6.996 \\
\hline$P_{\text {fake }}=0.2, K=5$ & 8.29 & 7289 & 7.143 \\
\hline$P_{\text {fake }}=0.3, K=5$ & 11.59 & 10591 & 7.269 \\
\hline$P_{\text {fake }}=0.1, K=6$ & 5.11 & 3425 & 7.127 \\
\hline$P_{\text {fake }}=0.2, K=6$ & 9.53 & 7111 & 7.266 \\
\hline$P_{\text {fake }}=0.3, K=6$ & 13.35 & 10287 & 7.323 \\
\hline
\end{tabular}

TABLE II

BFP TRANSMISSION OVERHEAD

The overhead cost of the fractal propagation protocol is shown in Table II. The transmission overhead (TO) is again the ratio of total number of packet transmissions to that with shortest single path transmission from a fixed source to a fixed destination 13 hops away. The number of different fake paths produced by fake packet propagation and the mean entropy values are also shown in the same table. For the shortest single path case, the entropy computed is 3.985 .

Obviously as $P_{\text {fake }}$ is increased and $K$ is increased, the overhead will be significantly increased. Even $P_{\text {fake }}=0.3$ and $K=5$ results in a high transmission overhead (TO) of $1159 \%$, but the anonymity and unlinkability is poor. The mean 
entropy values increase but provide no real information about privacy.

In comparison with fractal propagation, the unlinkability with random walk is better when $P_{r}$ is decreased. As illustrated in Fig. 2 the series of local maxima nodes tend to be irregular which make it harder for the global attacker to follow the traffic flow precisely.

\section{SECLOUD}

Next we test SECLOUD under identical circumstances as random walk and fractal propagation, with and without fake clouds.

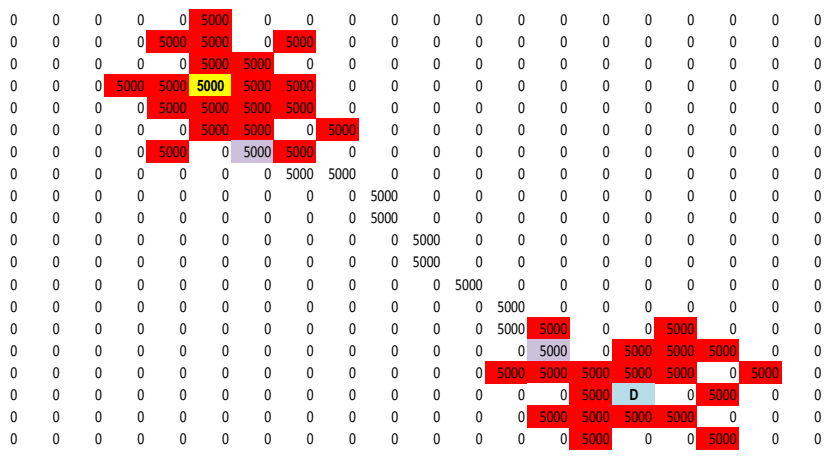

Fig. 8. SECLOUD with single path - marked nodes

First we consider a single delegated source and single delegated destination (gray cells) in Fig. 8. A cloud size $B<$ $4 k(k+1)$ was chosen in the case of grid networks where $k$ is the selected random hop distance from the source/destination. With $k=3$, we picked $B=20$ for this simulation. As it was designed to perform, two irregular clouds of identical broadcasting nodes of 5000 packets (highlighted in red) are formed around the source and destination. This will ensure the location privacy of the source and destination among their cloud regions, since the source could be any node inside the cloud, i.e., not necessarily at the center or the edge. The probability for a global attacker to guess the location of the source will be $1 /$ total number of nodes in both clouds. Note that the size of the clouds $B$ can be adjusted by the SECLOUD protocol, which means that the anonymity can be controlled. The linkability will not lead the attacker to the real source or destination, but only to the irregular cloud broadcasting region.

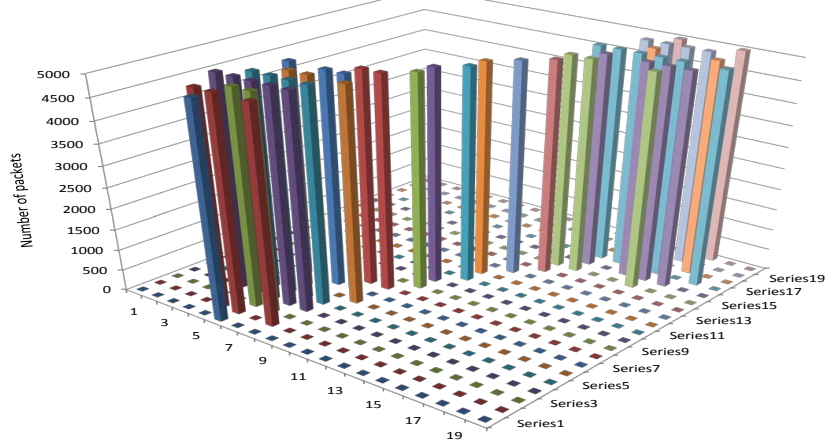

Fig. 9. SECLOUD with single path - 3-D Graph
Fig. 9 shows no local or global maxima in the network and confirms from what we have concluded from Fig. 8.

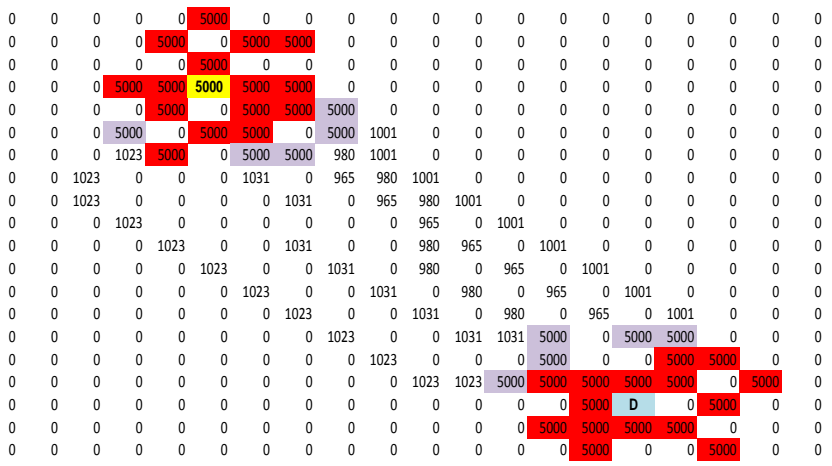

Fig. 10. SECLOUD with multiple paths - marked nodes

In Fig. 10, we show a sample result for multiple delegated sources and delegated destinations. Fig. 10 again shows two irregular clouds of nodes broadcasting 5000 packets (highlighted in red) formed around the source-side and destinationside. The real source sends 5000 packets using five delegated source-destination pairs (highlighted in gray), which also mean there are 5 transmission paths. Although using multiple paths is more complex to manage and setup, it has two advantages compared to single path. In the case where an attacker is resident on a path, using several disjoint paths will explicitly avoid that attacker. Moreover, using multiple paths will distribute the load of broadcasting the packets by intermediate nodes on those paths. The multiple paths also increase the unlinkability of the real source-destination pair. The anonymity level is similar to the case of SECLOUD with single path, as described previously.

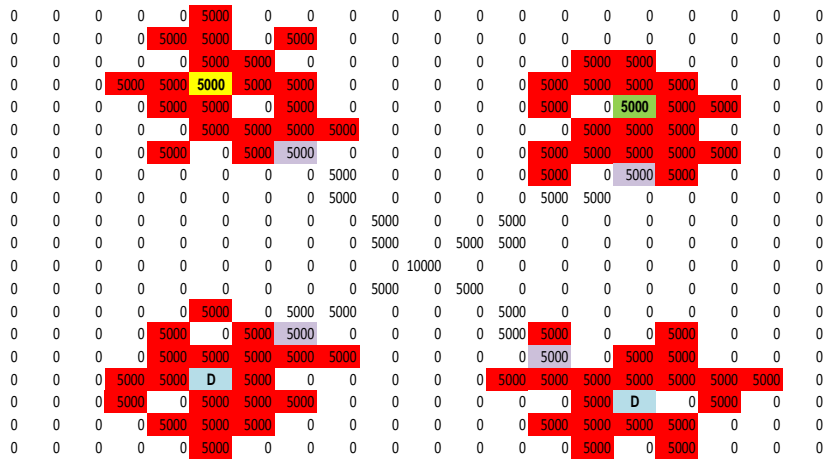

Fig. 11. SECLOUD with single path and fake clouds

Fig. 11 shows sample results for SECLOUD (single path with a fake source-destination pair). Compared to the previous case without a fake cloud, the attacker now not only needs to guess the location of the source within a cloud, but it also has to select the correct cloud and the guess the real source within it. The anonymity level in this case is doubled with one fake source-destination, and the unlinkability of real sourcedestination is higher. Moreover, it is not clear which cloud is the source and which is the destination.

In Fig. 12, we show a sample result with multiple paths and one fake source-destination pair. In addition to having all 


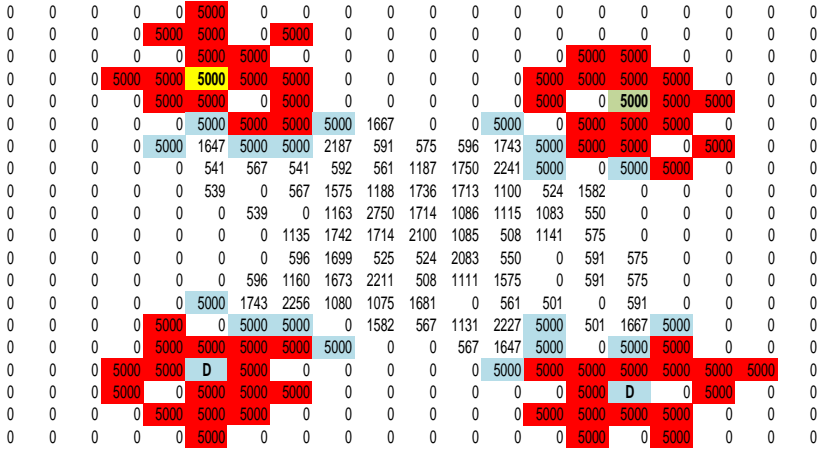

Fig. 12. SECLOUD with multi-path and fake clouds

the benefits of the previous approaches, this approach further increases the unlinkability between the real source-destination pair.

\section{Summary of Results and Comparison}

\begin{tabular}{|l|c|l|}
\hline Protocol & TO & Anonymity Level \\
\hline SECLOUD single path & 3.85 & $1-B^{-1}$ \\
\hline SECLOUD multipaths & 3.82 & $1-B^{-1}$ \\
\hline SECLOUD single paths w. fake & 7.69 & $1-[(\# \text { of cloud pairs }) B]^{-1}$ \\
\hline SECLOUD multipaths w. fake & 7.53 & $1-[$ (\# of cloudpairs $) B]^{-1}$ \\
\hline
\end{tabular}

TABLE III

SECLOUD RESULTS SUMMARY

The overhead performance and anonymity level of several cases of SECLOUD from simulations are shown in Table III. Using fake source-destination pairs will approximately double the overhead compared to the case without using any fake source-destination. As we increase the size of the cloud, the anonymity will increase but the overhead also will increase.

Compared to fractal propagation where the minimum overhead is $475 \%$, SECLOUD adds a fixed lower overhead as shown in Table III but provides better seclusion for both, source and destination nodes. The random walk protocol has an average overhead of $234 \%$ which is less that of SECLOUD. Combining random walk with cloud creation is a possibility under consideration.

In all the previous simulation results shown, we have used a grid network. However, for a random network in which the $\mathrm{X}$ and $\mathrm{Y}$ position of each node is independently and randomly using a uniform randomly distributed variable, the results of SECLOUD, Fractal and Random walk will all behave in a relatively similar manner. For brevity, we have not included the results in this paper.

Protecting privacy in the single source-destination case is more difficult compared to multiple pairs of sourcedestination. In the case of more than one real sourcedestination pairs, SECLOUD will have more pairs of clouds. If the attacker is targeting a specific source-destination, then the task of the attacker is also to select the correct sourcedestination from the correct cloud region. Note that to achieve privacy with more source-destination pairs, the sources have to start transmission roughly at the same time. Otherwise, a global attacker can monitor the network at time $T 1$ when the first source-destination is activated and then monitor the network at time $T 2$ when the second source-destination is activated.

\section{Conclusions}

In this work, we presented a protocol to address the problem of source-destination privacy in the presence of a global and powerful attacker with network-wide traffic knowledge. We compare it with fractal propagation and random walk and show that it performs better than fractal propagation and favorably with random walk in terms of overhead, anonymity, and unlinkability.

\section{REFERENCES}

[1] A. A. Nezhad, A. Miri, and D. Makrakis, "Location privacy and anonymity preserving routing for wireless sensor networks," Comput. Netw., vol. 52, no. 18, pp. 3433-3452, 2008.

[2] J. Deng, R. Han, and S. Mishra, "Decorrelating wireless sensor network traffic to inhibit traffic analysis attacks," Elsevier Journal of Pervasive and Mobile Computing on Security in Wireless Mobile Computing Systems, vol. 2, no. 2, pp. 159-186, 2006.

[3] K. Mehta, D. Liu, and M. Wright, "Location privacy in sensor networks against a global eavesdropper," in Proc. of IEEE ICNP, 2007.

[4] Y. Yang, M. Shao, S. Zhu, B. Urgaonkar, and G. Cao, "Towards event source unobservability with minimum network traffic in sensor networks," in Proc. of ACM WiSec, 2008.

[5] Y. Jian, S. Chen, Z. Zhang, and L. Zhang, "A novel scheme for protecting receiver's location privacy in wireless sensor networks," IEEE Trans. on Wireless Comm., vol. 7, no. 10, pp. 3769-3779, October 2008.

[6] Y. Xi, L. Schwiebert, and W. Shi, "Preserving source location privacy in monitoring based wireless sensor networks," in Proc. of SSN, 2006.

[7] P. Kamat, Y. Zhang, W. Trappe, and C. Ozturk, "Enhancing sourcelocation privacy in sensor network routing," in Proc. IEEE ICDCS, 2005.

[8] M. Shao, Y. Yang, S. Zhu, and G. Cao, "Towards statistically strong source anonymity for sensor networks," in Proc. of IEEE INFOCOM, 2008.

[9] C. Ozturk, Y. Zhang, W. Trappe, and M. Ott, "Source-location privacy for networks of energy-constrained sensors," in Proc. of IEEE SEUS, 2004.

[10] B. Hoh and M. Gruteser, "Protecting location privacy through path confusion," in Proc. of SECURECOMM, 2005.

[11] M. Gruteser and D. Grunwald, "Enhancing location privacy in wireless lan through disposable interface identifiers: a quantitative analysis," Mob. Netw. Appl., vol. 10, no. 3, pp. 315-325, 2005.

[12] H. Chan, A. Perrig, and D. Song, "Random key predistribution schemes for sensor networks," in Proc. of IEEE SSP, 2003.

[13] W. Du, J. Deng, Y. S. Han, and P. K. Varshney, "A key predistribution scheme for sensor networks using deployment knowledge," IEEE Trans. on Dependable and Secure Comp., vol. 3, no. 1, pp. 62-77, 2006.

[14] Y. Zhang, W. Liu, and W. Lou, "Anonymous communications in mobile ad hoc networks," in Proc. of IEEE INFOCOM, 2005.

[15] J. Kong, X. Hong, and M. Gerla, "An identity-free and on-demand routing scheme against anonymity threats in mobile ad hoc networks," IEEE Trans. on Mob. Comp., vol. 6, no. 8, pp. 888-902, 2007.

[16] R. Shokri, M. Yabandeh, and N. Yazdani, "Anonymous routing in manet using random identifiers," in Proc. of the Sixth International Conference on Networking, 2007.

[17] R. Lu, Z. Cao, L. Wang, and C. Sun, "A secure anonymous routing protocol with authenticated key exchange for ad hoc networks," Comput. Stand. Interfaces, vol. 29, no. 5, pp. 521-527, 2007.

[18] A. Boukerche, K. El-Khatib, L. Xu, and L. Korba, "Sdar: a secure distributed anonymous routing protocol for wireless and mobile ad hoc networks," in Proc. of IEEE LCN, 2004.

[19] D. Huang, "Traffic analysis based unlinkability measure for ieee 802.11b-based communication systems," in Proc. of ACM WiSe, 2006.

[20] F. Kuhn and A. Zollinger, "Ad-hoc networks beyond unit disk graphs," in Proc. of the fifth international workshop on Foundations of mobile computing, 2003.

[21] J. Canny, "A computational approach to edge detection," IEEE Transactions on Pattern Analysis and Machine Intelligence, vol. 8, pp. 679-714, November 1986. 